\title{
On the Evaluation of the Mechanical Behaviour of Structural Glass Elements
}

\author{
S. Costa ${ }^{1}$, M. Miranda ${ }^{1}$, H. Varum ${ }^{1, a}$ and F. Teixeira-Dias ${ }^{2, b}$ \\ ${ }^{1}$ Civil Engineering Department, University of Aveiro, 3810-193 Aveiro, Portugal \\ ${ }^{2}$ Mechanical Engineering Department, University of Aveiro, 3810-193 Aveiro, Portugal \\ ${ }^{a}$ hvarum@civil.ua.pt, ${ }^{b} \mathrm{ftd} @ m e c . u a . p t$
}

Keywords: Elastic modulus, Tensile strength, Ultimate curvature, Laminated glass, Float glass, Tempered glass

\begin{abstract}
Glass can be considered to be a high-technology engineering material with a multifunctional potential for structural applications. However, the conventional approach to the use of glass is often based only on its properties of transparency and isolation. It is thus highly appropriate and necessary to study the mechanical behaviour of this material and to develop adequate methods and models leading to its characterisation. It is evident that the great potential of growth for structural glass applications is an important opportunity of development for the glass industry and the building/construction sectors. The work presented in this paper is a reflection of this conclusion. The authors shortly present the state-of-the-art on the application of glass as a structural element in building and construction, and refer to other potential fields of application and available glass materials. The experimental procedures and methods adopted in three-point bending tests performed on $500 \times 100\left[\mathrm{~mm}^{2}\right]$ float, laminated and tempered glass specimens with thicknesses between 4 and $19 \mathrm{~mm}$ are thoroughly described. The authors evaluated the mechanical strength and stiffness of glass for structural applications. This work contributes to a deeper knowledge of the properties of this material.
\end{abstract}

\section{Introduction}

Glass is a material highly popular in modern architecture mostly due to its essential properties of transparency, isolation and durability. However, its mechanical properties of strength, stiffness, chemical resistance and fire protection make it also attractive in several engineering applications.

The use of glass has long been limited to the isolation and definition of boundaries between different environments, such as between the interior and the exterior of a house. However, it is necessary to develop different approaches to this material because it has a great engineering potential. Nowadays, a reduced number of glass structural applications can be found. In order to fully explore the potential of this material, a deeper mechanical characterisation of glass is needed.

Although it is possible to use glass as a structural element in construction, there are many engineering questions needing to be answered. Most of these are related to stability, strength and production of structural glass. Additionally, there are not yet any construction standards and/or recommendations at national or international level, for structural glass.

In the work here presented, the authors define a set of procedures to determine the mechanical behaviour of different types of glass commonly used in construction. In this way, the work contributes to the development and calibration of specific analysis methods and models leading to the structural characterisation of glass.

\section{Experimental Procedure}

Float, tempered and laminated glass samples were tested at room temperature using a Shimadzu ${ }^{\mathrm{TM}}$ AG-50kNG universal testing machine. This testing equipment was specifically adapted to the threepoint bending procedure used in this work. Fig. 1(a) and Fig. 1(b) show a schematic representation 
of the testing procedure and a photograph of the equipment, respectively. The adopted load application rate was $4 \mathrm{~mm} \cdot \mathrm{min}^{-1}$.

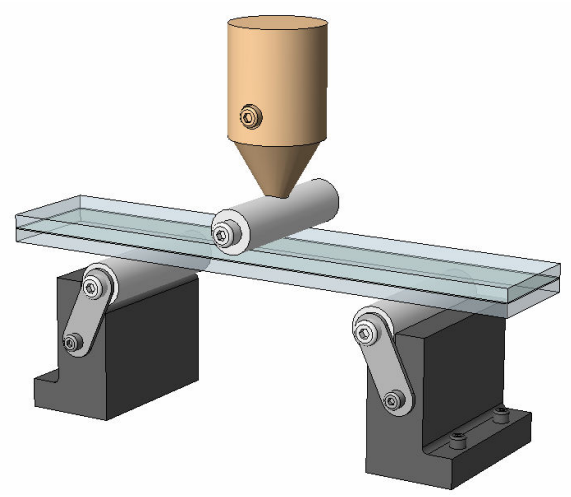

(a)

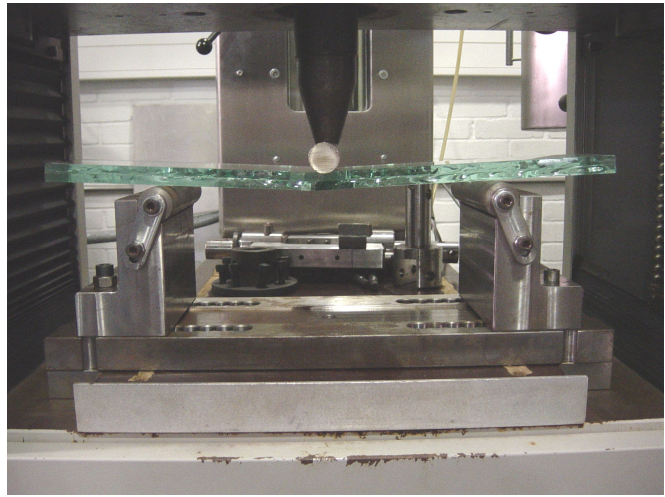

(b)

Figure 1: Experimental setup: (a) schematic representation of the testing procedure and (b) photograph of the Shimadzu ${ }^{\mathrm{TM}}$ AG-50kNG testing machine.

Three-point bending tests were performed on glass samples $100 \mathrm{~mm}$ wide and $500 \mathrm{~mm}$ long. The thicknesses for each type of glass studied are summarized in Table 1. Five samples were used for each glass type/thickness. These samples had no particular edge of surface treatment except for the tempered glass. The thickness of the polyvinyl butyral (PVB) interlayer in the laminated glass is $0.38 \mathrm{~mm}$ in all samples.

Table 1 - Thicknesses of the tested float, tempered and laminated glass specimens.

\begin{tabular}{ccccccccc}
\hline Thickness [mm] & $\mathbf{3}$ & $\mathbf{4}$ & $\mathbf{5}$ & $\mathbf{6}$ & $\mathbf{8}$ & $\mathbf{1 0}$ & $\mathbf{1 2}$ & $\mathbf{1 9}$ \\
\hline Float & & $\checkmark$ & & $\checkmark$ & & $\checkmark$ & & $\checkmark$ \\
Tempered & & $\checkmark$ & & $\checkmark$ & & $\checkmark$ & & $\checkmark$ \\
Laminated & 3.1 .3 & & 5.1 .5 & & 8.1 .8 & 10.1 .10 & 12.1 .12 & \\
\hline
\end{tabular}

\section{Results and Discussion}

The behaviour of the three types of glass for the $10 \mathrm{~mm}$ thickness samples is shown in Fig. 2. Note that for the tests performed on laminated glass this corresponds to an equivalent thickness $e^{\prime}=2^{(1 / 3)} e$, determined considering that the PVB interlayer does not exits. The actual specimen has two glass panes with $8 \mathrm{~mm}$ each (8.1.8 samples). Laminated glass exhibits a clearly distinct behaviour from that of float and tempered glass. The PVB interlayer in the laminated specimens has a significant influence on the behaviour of the material. Additionally, the fracture of the two glass panes is almost simultaneous for equivalent thicknesses below $10 \mathrm{~mm}$. However, above this limit value, the two glass panes fracture separately because sufficient strength is kept by the glass system to resist further to the applied load.

As expected, the elastic modulus (Young's modulus) is almost constant with the thickness for both float and tempered glass (see results in Fig. 3). The slight reduction with thickness shown in these results can be explained by the eventual change in the chemical composition of the material along the thickness, as a consequence of the fabrication process.

For float and tempered glass, the evolution in the elastic modulus is similar and approximately constant until the thickness of $10 \mathrm{~mm}$. However, for higher thickness values, there is an evident change in the relative mechanical behaviour of these two types of glass. Tempered glass exhibits higher elastic modulus values which are approximately $20 \%$ higher than those of the float glass (see the results in Table 2). This behaviour is expected and it is due to the fact that tempered glass is submitted to a tempering stage during the fabrication process. This thermal treatment induces compressive stresses in the surface of the glass panes and tension in the interior, which constitutes a 
favourable pre-stressing action. Therefore, the failure load corresponds to the external pre-tension stress plus the material tension capacity. The mechanical behaviour efficiency of the tempered glass depends on the glass thickness.

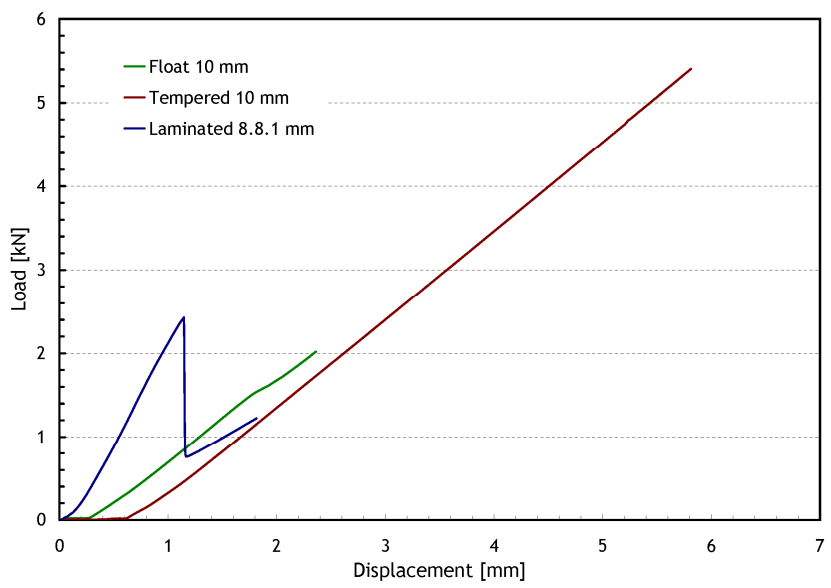

Figure 2: Example stress-strain behaviour for the float, tempered and laminated glass specimens.

The mechanical behaviour of laminated glass is distinct from the other tested glass types. The elastic modulus significantly decreases with the increase of the equivalent pane thickness. There are several possible explanations for this particular behaviour. Some lie in the fact that the thickness of the PVB interlayer is the same for all specimens, independent of the panes' thickness. Consequently, for the higher thickness values, the relative influence of the PVB layer on the behaviour of the glass system is reduced and the laminated glass system loses the monolithic behaviour guaranteed by the PVB layer, assuming a layered behaviour. One additional explanation is based on the fact that the mechanical properties of the glass panes are dependent of the chemical composition along thickness of the specimen.

The elastic modulus of the thinnest laminated glass system (system 3.1.3, corresponding to two $3 \mathrm{~mm}$ glass panes with a $0.38 \mathrm{~mm}$ PVB interlayer) is higher than that of the thickest $(19 \mathrm{~mm})$ float glass. These results are summarised in Table 2.

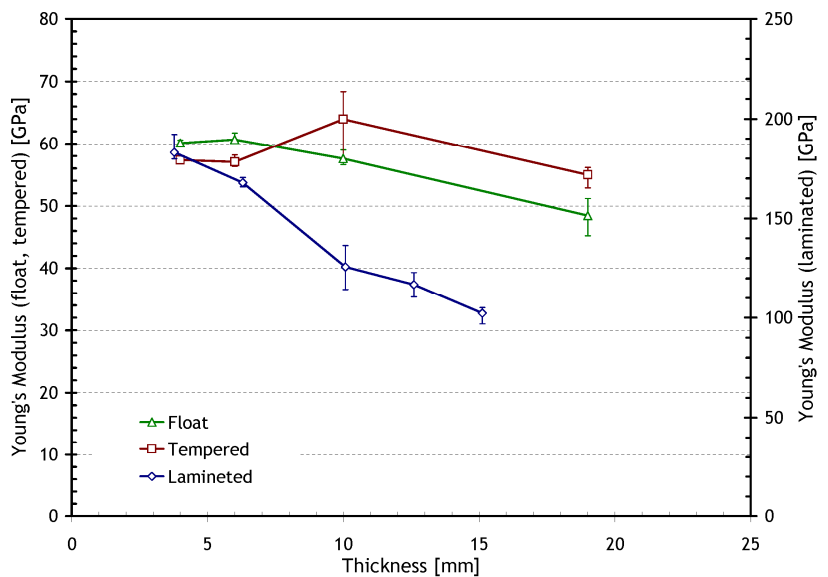

Figure 3: Elastic modulus as a function of thickness for float, tempered and laminated glass specimens.

The evolution of the failure strain with thickness for all glass specimens is shown in the results in Fig. 4. Failure strain significantly decreases with the thickness of the specimens for all glass types. For the higher thickness values, this behaviour is not so evident probably because the fracture of the two glass panes happens simultaneously. The visco-plastic behaviour of the PVB interlayer increases the deformability of the laminated glass system. A significant dispersion in the values of the failure strain and tensile strength can be noticed for samples with the same dimensions and test conditions (see Fig. 4 and Fig. 5). This dispersion is probably due to the influence of the edge 
finishing and surface imperfections resulting from the fabrication process. The dispersion is more significant for float and tempered glass.

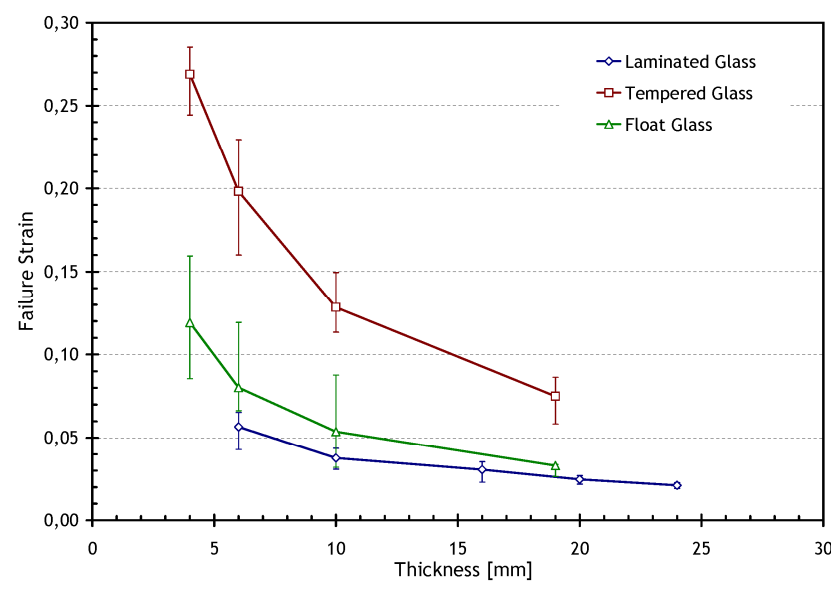

Figure 4: Failure strain versus thickness for float, tempered and laminated glass specimens.

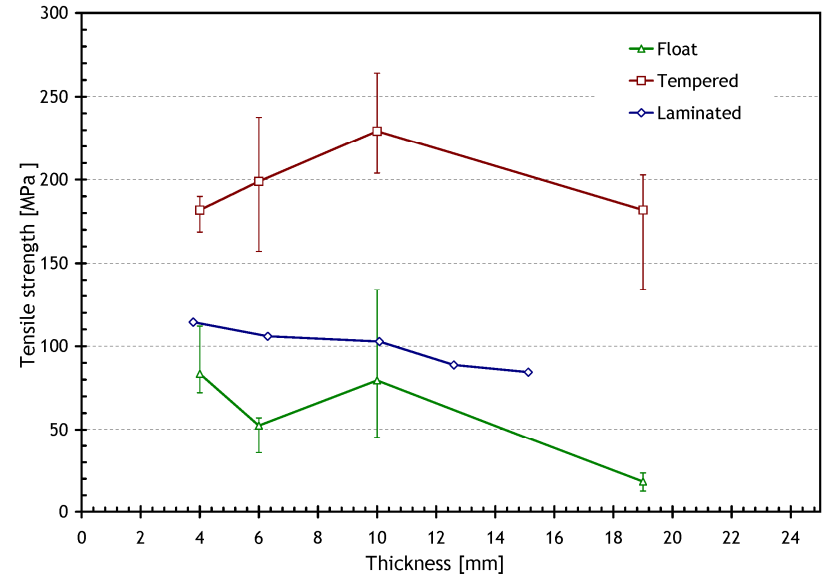

Figure 5: Tensile strength as a function of thickness for float, tempered and laminated glass specimens.

Table 2 - Average values for the elastic modulus $(E)$, tensile strength $(\sigma)$ and failure strain $\left(\varepsilon_{\mathrm{f}}\right)$.

\begin{tabular}{ccccc}
\hline & Thickness $[\mathbf{m m}]$ & $\boldsymbol{E}[\mathbf{G P a}]$ & $\boldsymbol{\sigma}[\mathbf{M P a}]$ & $\boldsymbol{\varepsilon}_{\mathrm{f}}$ \\
\hline \multirow{4}{*}{ Float } & 4 & 60.12 & 83.57 & 0.12 \\
& 6 & 60.70 & 52.56 & 0.08 \\
\hline \multirow{3}{*}{ Tempered } & 10 & 57.58 & 79.57 & 0.05 \\
& 19 & 48.44 & 18.28 & 0.03 \\
\hline \multirow{3}{*}{ Laminated } & 4 & 57.36 & 181.78 & 0.27 \\
& 10 & 57.04 & 198.95 & 0.20 \\
& 19 & 63.97 & 229.35 & 0.13 \\
& 3.1 .3 & 54.97 & 181.82 & 0.07 \\
\hline & 5.1 .5 & 183.01 & 114.36 & 0.057 \\
\hline & 10.1 .8 & 167.77 & 105.94 & 0.038 \\
\hline & 12.1 .12 & 125.75 & 102.79 & 0.031 \\
\hline & & 116.86 & 88.79 & 0.025 \\
\hline
\end{tabular}

In terms of fracture modes, float glass breaks in large pieces and with straight and smooth fracture surfaces (seen Fig. 6). Tempered glass breaks into very small and irregular pieces (see Fig. 7). However, laminated glass breaks in convergent straight lines and the fragments remain bonded to 
the interlayer PVB. This justifies the use of laminated glass as security glass. Smaller thickness of laminated glass systems exhibit a higher deformation capacity, as shown in the results in Fig. 4 and comparing Fig. 8(a) and Fig. 8(b). These differences in the fracture modes are mostly due to: (i) the differences in chemical composition and (ii) the tensile-compression residual stress states induced along the thickness of the specimens.

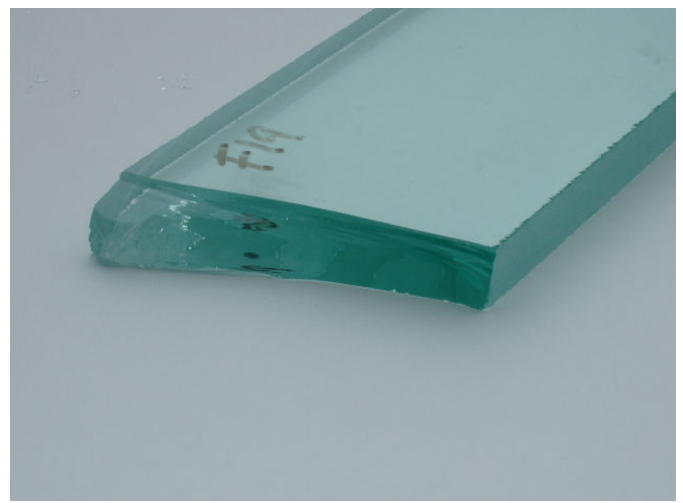

Figure 6: Crack pattern of float glass.

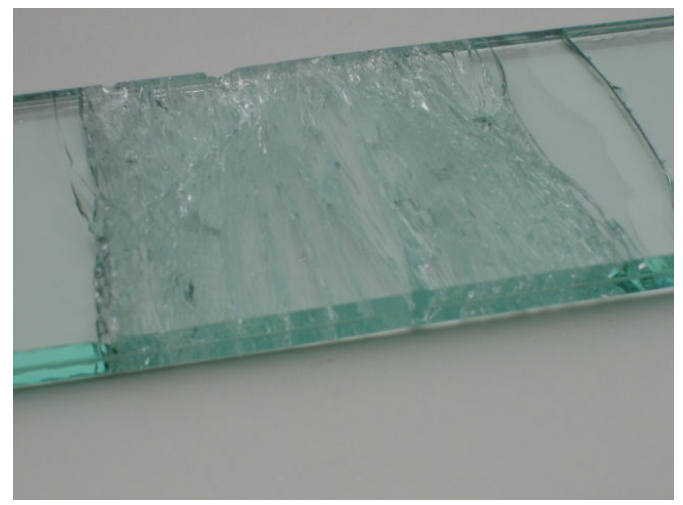

(a) Laminated system 5.1.5.

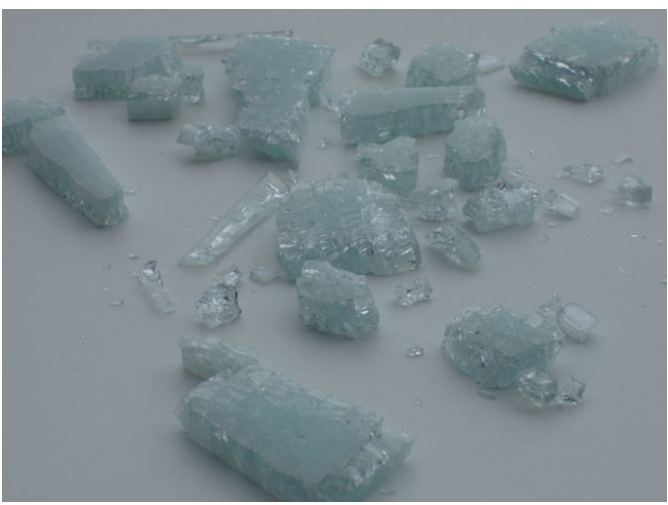

Figure 7: Crack pattern of tempered glass.

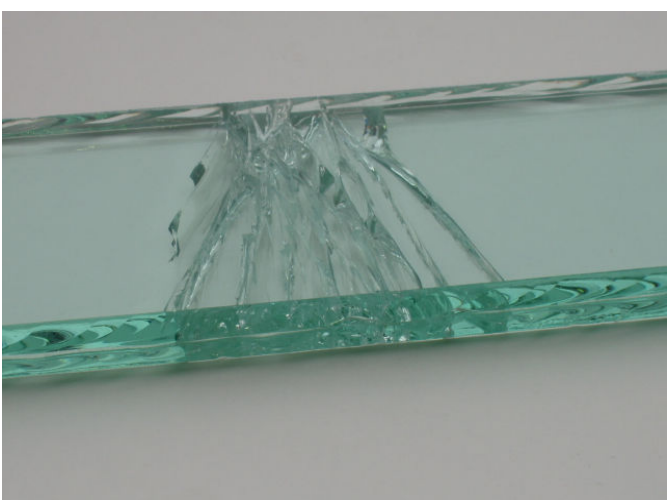

(b) Laminated system 10.1.10.

Figure 8: Crack pattern for the laminated glass samples.

\section{Acknowledgements}

Authors would like to thank Eng ${ }^{\mathrm{o}}$ António Bastos, from the Department of Mechanical Engineering of the University of Aveiro for the support given during the experimental tests. Authors also thank Covipor-La Veneciana de Saint Gobain for providing the glass samples.

\section{References}

[1] A.S. Redner and B.R. Hoffman: Detection of tensile stresses near edges of laminated and tempered glass (Glass Processing Days p.589-591, 2001).

[2] R. Hess: Material Glass (Structural Engineering International, Glasconsult, Utikon, Suiça).

[3] SAINT GOBAIN GLASS: Manual do Vidro, 2000.

[4] J. Belis and R. Impe: The Invisible Building: Advanced Research on Glass Structures, (Department of Structural Engineering, Ghent of University).

[5] A. Luible and M. Crisinel: Buckling Strength of Glass Elements in Compression, (Structural Engineering International, Glasconsult, Utikon, Suiça).

[6] R. Kasper and, G. Sedlacek: Structural Use of Glass Beams, (Institute of Steel Construction of the University of Aachen, RWTH Aachen).

[7] Y. Rodichev: Design and Mechanical Properties of New Laminated Glass Materials and Structural Elements, (Institute for Problems of Strength). 